\title{
Detecting overlapping protein complexes in weighted PPI network based on overlay network chain in quotient space
}

Jie Zhao and Xiujuan Lei

From 2018 International Conference on Intelligent Computing (ICIC 2018) and Intelligent Computing and Biomedical Informatics (ICBI) 2018 conference

Wuhan and Shanghai, China. 15-18 August 2018, 3-4 November 2018

\begin{abstract}
Background: Protein complexes are the cornerstones of many biological processes and gather them to form various types of molecular machinery that perform a vast array of biological functions. In fact, a protein may belong to multiple protein complexes. Most existing protein complex detection algorithms cannot reflect overlapping protein complexes. To solve this problem, a novel overlapping protein complexes identification algorithm is proposed.

Results: In this paper, a new clustering algorithm based on overlay network chain in quotient space, marked as ONCQS, was proposed to detect overlapping protein complexes in weighted PPI networks. In the quotient space, a multilevel overlay network is constructed by using the maximal complete subgraph to mine overlapping protein complexes. The GO annotation data is used to weight the PPI network. According to the compatibility relation, the overlay network chain in quotient space was calculated. The protein complexes are contained in the last level of the overlay network. The experiments were carried out on four PPI databases, and compared ONCQS with five other state-of-the-art methods in the identification of protein complexes.

Conclusions: We have applied ONCQS to four PPI databases DIP, Gavin, Krogan and MIPS, the results show that it is superior to other five existing algorithms MCODE, MCL, CORE, ClusterONE and $C O A C H$ in detecting overlapping protein complexes.
\end{abstract}

Keywords: Protein complexes, Gene ontology, Quotient space, Granular computation, Clustering

\section{Introduction}

Analyzing the mechanism of proteins is crucial for understanding the function of cell machinery and explaining biological processes [1]. Proteins often bind together to form complexes to carry out their biological functions $[2,3]$. A protein complex is a molecular group of two or more functionally related proteins assembled via multiple protein interactions [4]. Detecting protein complexes has great significance in biology and proteomics [5]. In the early stage of protein complex research, the

\footnotetext{
* Correspondence: xjlei@snnu.edu.cn

School of Computer Science, Shaanxi Normal University, Xi'an 710119 Shaanxi, China
}

protein complexes were found mainly through biological experiments methods, such as RNA interference, conditional gene knockout, single gene knockout and Coimmunoprecipitation $[6,7]$. However, these methods are costly and time-consuming.

The high throughput techniques have generated a large amount of protein related data. In 2001, Legrain et al. [8] described the protein-protein interactions (PPI) as an undirected graph $\mathrm{G}(V, E)$, where the point set $V$ represents protein nodes and the edge set $E$ represents protein-protein interactions. This idea transforms largescale protein-protein interaction data into network structure, which triggered scholars to recognize protein complexes based on the topological properties of protein 
networks. In 2003, Bader and Hogue [9] proposed MCODE method which is a local-search method to detect protein complexes based on the proteins' connectivity values in PPI network. In 2006, Gavin et al. [10] demonstrated that protein complexes was made up of core and additional attachment proteins or protein modules. According to the core-attachment structure of protein complexes, Leung et al. [11] designed CORE algorithm which calculated the $p$-value for all pairs of proteins to detect cores. Wu et al. [12] proposed $\mathrm{COACH}$ algorithm which detected dense subgraphs as cores. In 2009, Liu et al. [13] presented a method called CMC which identified protein complexes based on maximal cliques. In fact, a protein may belong to multiple protein complexes, and there may be overlaps between protein complexes. In 2012, NepusZ et al. developed a clustering algorithm ClusterONE [14] to detect overlapping protein complexes. Recently, attributed network embedding methods have be proved to be remarkably effective in generating vector representations for nodes in the network [15]. $\mathrm{Xu}$ et al. designed a method GANE to predict protein complexes based on Gene Ontology attributed network embedding [15].

Some classical clustering algorithms such as Markov Clustering (MCL) [16] and swarm intelligence optimization algorithm $[17,18]$ were also developed to detect protein complexes. Lei et al. [19] proposed FMCL clustering model based on Markov clustering in which automatically adjusted the parameters by introducing the firefly algorithm. Wang et al. [4] developed a heuristic graph clustering algorithm called HGCA based on multiple topological characteristics.

In recent years, quotient space theory has been applied to cluster. Zhang [20] defined the fuzzy equivalence relation and stratified hierarchical structure, and established the fuzzy granular computing model in quotient space in order to solve the uncertain problem. Xu [21] proposed fuzzy clustering method based on Gaussian function. The method, with the nature of the distance metric spaces, merged the individual particles in information synthesis way for clustering results. Cluster analysis method [22] based on fuzzy similarity relations and normalized distance is proposed to solve data structure analysis of complex systems. The conclusion is suitable for the complicated systems.

In this study, a new clustering algorithm based on overlay network chain in quotient space, marked as ONCQS, was proposed to detect overlapping protein complexes in weighted PPI networks. Firstly, the GO annotation data is used to weight the PPI network. Then, the maximal complete subgraph of the PPI network is found. The maximal complete subgraph of the current network is regarded as the node in the next layer of network. According to the compatibility relation, the overlay network chain in quotient space is calculated, the protein complexes are contained in the last layer of the overlay network. The algorithm ONCQS is tested on four well-known PPI databases DIP [23], Gavin [10], Krogan [24] and MIPS [25]. The simulation results illustrate that ONCQS algorithm has a higher performance and outweighs than other five algorithms in mining protein complexes.

\section{Methods}

\section{Constructing weighted PPI network}

It is inaccurate to mine protein complexes directly in PPI networks because the data produced by highthroughput experiments contain a high rate of false positive and false negative interactions [26, 27].To address this problem, some scholars integrate protein biologic data such as gene expression data, subcellular localization data, GO annotation data $[28,29]$ to increase the reliability and accuracy of data. A protein complex is a group of two or more associated polypeptide chains. Different polypeptide chains may have same functions, so we integrate GO annotation data to measure the interactions. If two interacted proteins $v_{i}$ and $v_{j}$ have more common GO annotations, their functions are more similar and their interaction is believed to be more believable. The weight between protein $v_{i}$ and $v_{j}$ is defined as follows:

Table 1 Pseudo code of maximum complete subgraph

Algorithm 1: maximal complete subgraphs
Input: The weighted PPI network: $G(V, E)$
The adjacency matrix of $G: A\left(a_{i j}=a_{j i}\right)$
Output: The sets of maximal complete subgraphs: $M C S$
1. $M C S=\varnothing$
2. for each node $v_{i} \in V(i=1,2, \ldots n)$
3. $a_{i}=\left(a_{i l}, a_{i 2}, \ldots, a_{i n}\right)$
4. find the first component that is not equal to $0: a_{i i l}$
5. $M C S_{i}=\left(a_{i}, a_{i l}\right)$
6. if $\exists i 2 \neq i, i 1, a_{i i 2}=a_{i l i 2}$
7. $M C S_{i}=\left(a_{i}, a_{i l}, a_{i 2}\right)$
8. end if
9. Repeat $5-7$, find the common neighbors of nodes in $M C S_{i}$
10. $M C S_{i}=\left(a_{i}, a_{i l}, a_{i 2, \ldots,}, a_{i m}\right)$
11. end for
12. $M C S=\left\{M C S_{1,} M C S_{2, \ldots,} M C S_{n}\right\}$
13. return $M C S$




$$
W_{v_{i}, v_{j}}=\frac{\left|G O_{v_{i}} \cap G O_{v_{j}}\right|}{\min \left(\left|G O_{v_{i}}\right|,\left|G O_{v_{j}}\right|\right)}
$$

where $G O_{v_{i}}$ and $G O_{v_{i}}$ are the $\mathrm{GO}$ annotation set of node $v_{i}$ and $v_{j}$ respectively, $\left|G O_{v_{i}} \cap G O_{v_{j}}\right|$ represents the number of the same annotation between $G O_{v_{i}}$ and $G O_{v_{j}}$. Our previous research shows that the $W_{v_{i}, v_{j}}$ value is greater than 0.6 , and the effect is better [30]. If weight between protein $v_{i}$ and $v_{j}$ is less than 0.6, the interaction will be deleted in the PPI network. This preprocessing step can help us to filter out possible false positive interactions [31].

\section{Quotient space theory}

Granular computing is a simulation of global analysis ability of human beings. One of the basic characteristics in human problem solving is the ability to conceptualize the world at different granularities and translate from one abstraction level to the others easily, deal with them hierarchically. Human beings can solve problems in different sizes of granularity spaces. Different levels represent different granularity.

There are three main theories of granular computing, granular computing based on fuzzy logic [32], granular computing based on rough set and granular computing based on quotient space. Granularity analysis is in fact to analyze the quotient set.

Triple structure $(X, F, T)$ is used to represent the problem in the quotient space. Domain $X$ refers to universe of discourse, $F$ is the attribute set of $X, T$ is the structure of $X$. Define a relation $R$ for the universe of discourse $X$, construct corresponding quotient set $[X]$, quotient attribute set $[F]$, and quotient structure $[T]$, and then define the granularity coefficient to study the quotient space $([X],[F],[T])$. The relation $R$ can be equivalence relation or compatibility relation.

For the PPI network $\mathrm{G}, G=(X, F, T)$, domain $X$ refers to the protein nodes in PPI network.

\section{Overlay network chain in quotient space}

Given a network $G$, the maximum complete subgraph of the network is regarded as a cover according to the compatibility relation [33]. The pseudo code of the maximum complete subgraph algorithm is shown in Table 1 .

After the sets of all maximal complete subgraphs is solved. Then, maximal complete subgraphs are used as nodes, if two maximal complete subgraphs have common nodes, two corresponding nodes are defined to be

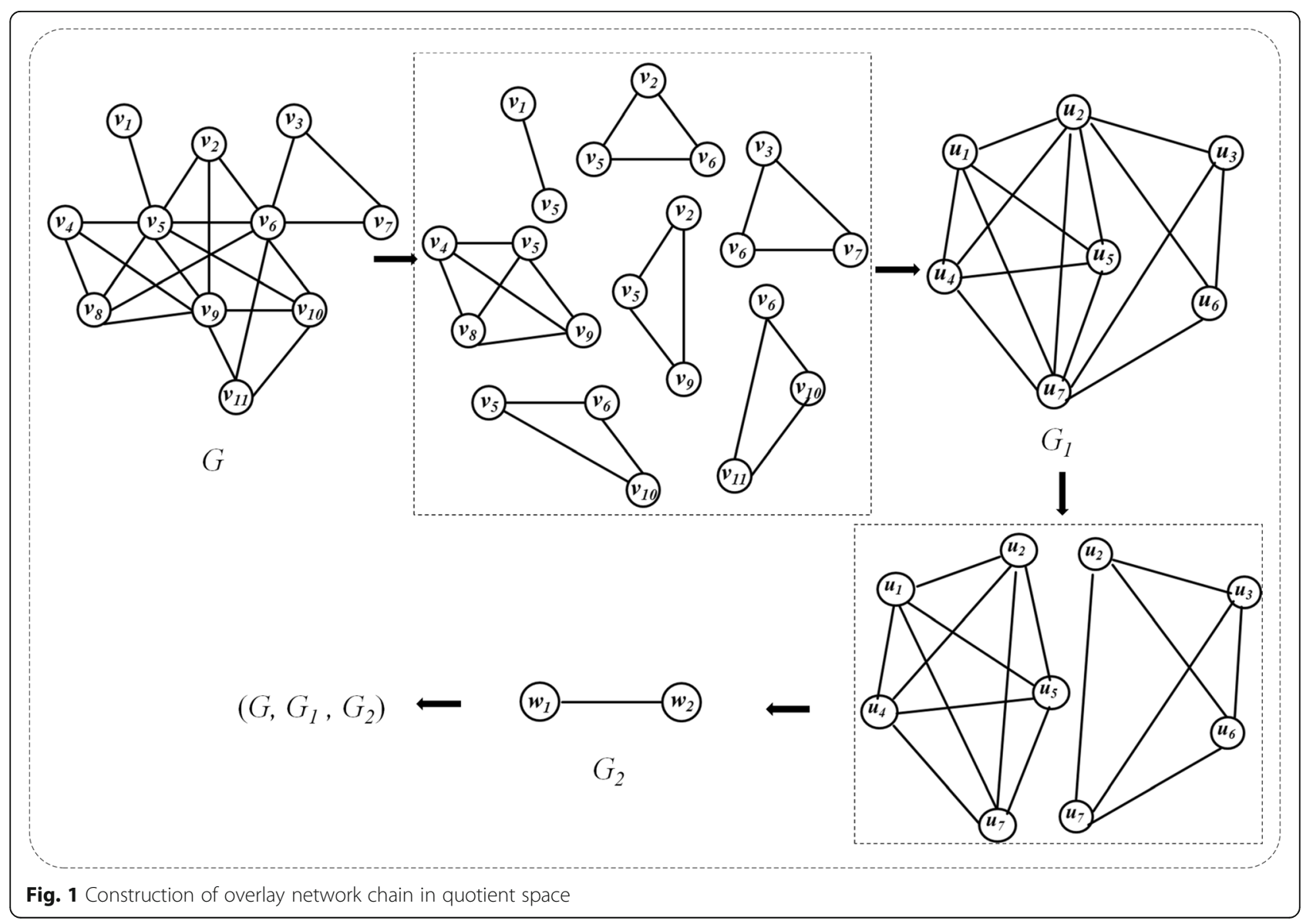


connected, the new network constructed is called the $1^{\text {st }}$ level overlay network of $G$ in quotient space, which is denoted as $G_{1}$. Figure 1 illustrates the construction of overlay network chain in quotient space. The network $G$ has 11 nodes $\left(v_{1}, v_{2}, v_{3}, v_{4}, v_{5}, v_{6}, v_{7}, v_{8}, v_{9}, v_{10}, v_{11}\right)$. There are 7 maximal complete subgraphs in the network $G$, so there are 7 nodes $\left(u_{1}, u_{2}, u_{3}, u_{4}, u_{5}, u_{6}, u_{7}\right)$ in the $1^{\text {st }}$ level overlay network $G_{1} \cdot u_{1}$ represents $\left(v_{1}, v_{5}\right), u_{2}$ represents $\left(v_{2}, v_{5}, v_{6}\right), u_{3}$ represents $\left(v_{3}, v_{6}, v_{7}\right), u_{1}$ and $u_{2}$ has common nodes $v_{5}, u_{2}$ and $u_{3}$ has common nodes $v_{6}$, so $u_{1}$ and $u_{2}$ are connected in $G_{1}, u_{2}$ and $u_{3}$ are connected in $G_{1}, u_{1}$ and $u_{3}$ have no common nodes, and there is no connection between them in $G_{1}$. Network $G_{1}$ has two complete subgraphs, the 2nd level overlay network $G_{2}$ has 2 nodes $\left(w_{1}, w_{2}\right) . w_{1}$ represents $\left(u_{1}, u_{2}, u_{4}\right.$, $\left.u_{5}, u_{7}\right), w_{2}$ represents $\left(u_{2}, u_{3}, u_{6}, u_{7}\right), w_{1}$ and $w_{2}$ has common nodes $\left(u_{2}, u_{7}\right)$, so $w_{1}$ and $w_{2}$ are connected in $G_{2}$. $G_{1}$ and $G_{2}$ are different levels of overlay network of $G$, $\left(G, G_{1}, G_{2}\right)$ is called overlay network chain.

Assuming that $G_{i}$ is the $i^{\text {th }}$ level overlay network of $G$, and $G_{i+1}$ is the 1st level overlay network of $G_{i}$, therefore, $G_{i+1}$ is the $(i+1)^{\text {th }}$ level overlay network of G. $\left(G, G_{1}, G_{2}, \ldots, G_{i}\right)$ is called overlay network chain in quotient space [34].

\section{The ONCQS main algorithm}

A new clustering algorithm ONCQS is developed to detect overlapping protein complexes in weighted PPI network using overlay network chain in quotient space. A protein may belong to multiple protein complexes. As shown in Fig. 2, two protein complexes elF3 complex and multi-elF complex in the CYC2008 benchmark have three overlapped proteins.

In overlay network $G_{i}$, each node represents a maximum complete subgraph of overlay network $G_{i-1}$. There may be repeated points and edges between maximal complete subgraphs. The protein complexes are contained in the last level of the overlay network. Each point can be regarded as a complex. So overlapping protein complexes can be found by using covering network. As shown in Fig. 1, in $G_{2}, w_{1}$ represents $\left(v_{1}, v_{2}, v_{4}, v_{5}, v_{6}, v_{8}, v_{9}, v_{10}\right), w_{2}$ represents $\left(v_{2}, v_{3}, v_{5}, v_{6}, v_{7}, v_{10}, v_{11}\right)$, they have four overlapped nodes.

In algorithm ONCQS, the static PPI network is usually described as an undirected graph $\mathrm{G}(V, E)$ which consists of a set of nodes $V$ and a set of edges $E$, the nodes $V$ represents the proteins and the edges $E=\left\{e\left(v_{i}, v_{j}\right)\right\}$ is the set of edges connecting two proteins $v_{i}$ and $v_{j}$. First, we use GO annotation data to weight the PPI network, and then construct multilevel overlay network. In overlay network theory, if two maximal complete subgraphs have common nodes, two corresponding nodes are defined to be connected. However, in ONCQS algorithm, formula 2 is used to

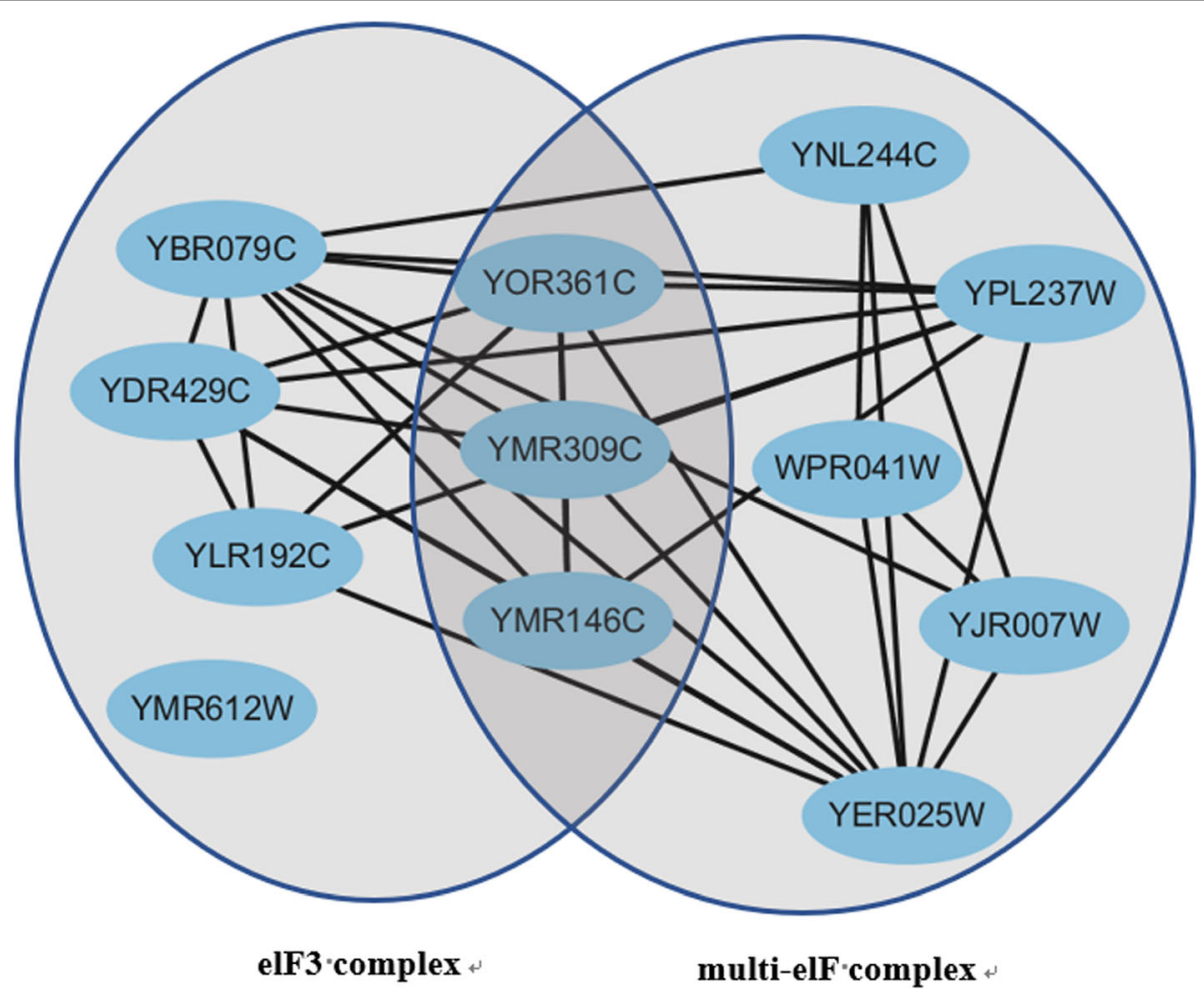

Fig. 2 An example of overlapping protein complexes 
measure the similarity of two maximal complete subgraphs $m c s_{i}$ and $m s c_{j}$.

$$
\operatorname{sim}\left(m c s_{i}, m c s_{j}\right)=\frac{\left|m c s_{i} \cap m c s_{j}\right|}{\left|m c s_{i} \cup m c s_{j}\right|}
$$

where $\left|m c s_{i} \cap m c s_{j}\right|$ is the number of the common nodes of $m c s_{i}$ and $m s c_{j},\left|m c s_{i} \cup m c s_{j}\right|$ is the summation of the nodes of $m c s_{i}$ and $m s c_{j}$. Only when $\operatorname{sim}\left(m c s_{i}, m c s_{j}\right)$ is great than the granularity coefficient $g c$, two corresponding nodes are defined to be connected in the next level overlay network. In $i^{\text {th }}$ level overlay network, if there is no maximal complete subgraph satisfying the similarity condition, the overlay network chain $\left(G, G_{1}, G 2, \ldots, G_{i}\right)$ can be obtained. The pseudo code of the ONCQS algorithm is shown in Table 2.

Table 2 Pseudo code of the ONCQS algorithm

Algorithm 2: ONCQS main function

Input: The weighted PPI network: $G(V, E)$

Parameter: granularity coefficient: $g c$

Output: The detected protein complex Complex

\section{Begin}

1. $P C=\varnothing$

2. Construct the overlay network $G_{i}\left(V_{i}, E_{i}\right)$ of the current network $G(V, E)$

3. Find the maximum complete subgraph sets $M C S_{G}$ of $G$;

4. $\forall m s c_{j} \in M C S_{G}$,

5. $m s c_{j} \in V i$

6. $\forall\left(m s c_{j}, m s c_{k}\right) \in M C S_{G}$

7. if $\operatorname{sim}\left(m c s_{j}, m c s_{k}\right)>g c$

8. $E i\left(m c s_{j}, m c s_{k}\right)=1$

9. end if

10. Overlay network $G_{i}\left(V_{i}, E_{i}\right)$ construction ended

11. if $\exists v_{i} \in G$ and $v_{i} \notin G i$

12. $v_{i} \in$ Complex

13. end if

14. $G(V, E)=G_{i}\left(V_{i}, E_{i}\right)$

15. Repeat 2-14, construct overlay network chain $\left(G(V, E), G_{l}\left(V_{l}, E_{l}\right), G 2\left(V_{2}, E_{2}\right), \ldots, G_{m}\left(V_{m}, E_{m}\right)\right)$

16. $\forall v \in V_{m}$

17. $v \in$ Complex $C$

18. Refinement procedure

19. return Complex

End
At this point, each node in $G_{i}$ represents a protein complex. Each node represents a maximal complete subgraph, so the proteins in the subgraph have high similarity and the similarity between the subgraphs is poor.

\section{Results and discussion}

The proposed ONCQS algorithm is implemented in Matlab R2015b and executed on a quad-core processor 3.30GHz PC with 8G RAM.

\section{Experimental data set}

In this study, the developed methods and computational analysis were applied to four PPI network, including DIP [23], Gavin [10], Krogan [24] and MIPS [25]. All the data used in this study are Saccharomyces cerevisiae protein data.

Protein-protein interactions data: After removing the noise, the self-interactions and the repeated interactions, DIP dataset (version of 20160114) included 5028 proteins and 22,302 interactions, Gavin dataset consists of 1430 proteins and 6531 interactions, Krogan dataset consists of 2674 proteins and 7075 interactions, the MIPS dataset included 4546 proteins and 12,319 interactions.

Gene Ontology data: The Saccharomyces cerevisiae GO annotation data was extracted from GO-slims dataset. GO-slims data are cut-down version of the GO ontologies [31]. GO-slim data provide GO terms to explain gene product feature in biological process (BP), molecular function (MF), cellular component (CC). we used GO slims to annotate PPI data. There are 7014 proteins in the GO annotation data. Proteins with GO annotation data cover $98.23 \%$ of proteins in the DIP dataset, $100 \%$ of proteins in Gavin, $99.89 \%$ of proteins in Krogan, 99.16\% of proteins in MIPS.

The standard protein complexes: CYC2008 [35] is used to evaluate clustering results of Saccharomyces cerevisiae, which includes 408 protein complexes. Detailed data intersection information of experimental data is shown in Table 3.

\section{Evaluation metrics}

The overlapping score $O S$ is used to evaluate the match quality of a predicted protein complex and standard protein complex.

$$
\mathrm{OS}(p c, s c)=\frac{\left|V_{p c} \cap V_{s c}\right|^{2}}{\left|V_{p c}\right| \times\left|V_{s c}\right|}
$$

where $V_{p c}$ and $V_{s c}$ denote the node sets of predicted protein complex $p c$ and standard protein complex $s c$, respectively. Usually we set the threshold for 0.2 [17]. If $O S(p c, s c)$ is greater than 0.2 , the predicted protein complex $p c$ is considered to match standard protein complex sc. $O S=1$ shows that the predicted protein complex is perfectly matched with the standard protein complex. 
Table 3 The data information of the experimental data

\begin{tabular}{lllll}
\hline Dataset & Number of node & Number of edge & Density & GO annotation data \\
\hline DIP & 5028 & 22,302 & 0.0018 & 4939 (98.23\%) \\
Gavin & 1430 & 6531 & 0.0064 & $1430(100 \%)$ \\
Krogan & 2674 & 7075 & 0.0020 & $2671(99.89 \%)$ \\
MIPS & 4546 & 12,319 & 0.0012 & 4508 (99.16\%) \\
\hline
\end{tabular}

Three commonly used metrics Precision, Recall and Fmeasure are used to measure the efficiency of the proposed ONCQS algorithm and evaluate the performance of the clustering results.

The Precision denotes the accuracy of the predicted protein complexes matched by the standard protein complexes, defined as follows:

$$
\text { Precision }=\frac{|m p c|}{|p c|}
$$

where $|p c|$ represents the number of predicted protein complexes, $|m p c|$ denotes the number of the predicted protein complexes matched by the standard protein complexes.

The Recall denotes the accuracy of the standard protein complexes matched by the predicted protein complexes, defined in the following eq. (5):

$$
\text { Recall }=\frac{|m s c|}{|s c|}
$$

where $|s c|$ represents the number of the standard protein complexes, $|m s c|$ denotes the number of the standard protein complexes matched by the predicted protein complexes.

The Precision and Recall describe the accuracy of the algorithm from different aspects. In order to consider these two indicators synthetically, the F-measure is defined as the harmonic mean of Precision and Recall. Fmeasure is defined as follows:

$$
\text { F-measure }=\frac{2 \times \text { Precision } \times \text { Recall }}{\text { Precision }+ \text { Recall }}
$$

\section{Parameter analysis}

The proposed algorithm ONCQS only has one parameter, granularity coefficient: $g c$. In overlay network, the similarity of two maximal complete subgraphs is greater than $g c$, we consider them connected in the next level overlay network. If the value of $g c$ is too small, the complexity of algorithm will increase. On the contrary, if the value of $g c$ is too large, the accuracy of the algorithm will decrease. It is significant to select the appropriate value of $g c$.

The experiments on four PPI databases with $g c$ from 0.1 to 0.9 were carried out to verify the influence of parameter

\begin{tabular}{|c|c|c|c|c|c|c|c|}
\hline Dataset & $g c$ & Precision & Recall & F-measure & $P C$ & Perfect & $A S$ \\
\hline \multirow[t]{9}{*}{$\overline{D I P}$} & 0.1 & 0.4199 & 0.7108 & 0.5279 & 874 & 62 & 5.18 \\
\hline & 0.2 & 0.4011 & 0.7206 & 0.5153 & 945 & 60 & 4.79 \\
\hline & 0.3 & 0.3571 & 0.7402 & 0.4818 & 1095 & 69 & 3.70 \\
\hline & 0.4 & 0.3561 & 0.8260 & 0.4976 & 1640 & 103 & 2.67 \\
\hline & 0.5 & 0.3521 & 0.8284 & 0.4942 & 1667 & 104 & 2.60 \\
\hline & 0.6 & 0.3470 & 0.8211 & 0.4878 & 1781 & 105 & 2.53 \\
\hline & 0.7 & 0.3499 & 0.8186 & 0.4902 & 1832 & 103 & 2.54 \\
\hline & 0.8 & 0.3530 & 0.8186 & 0.4933 & 1844 & 102 & 2.56 \\
\hline & 0.9 & 0.3530 & 0.8186 & 0.4933 & 1844 & 102 & 2.56 \\
\hline \multirow[t]{9}{*}{ Gavin } & 0.1 & 0.6581 & 0.4167 & 0.5103 & 310 & 38 & 7.99 \\
\hline & 0.2 & 0.6085 & 0.4265 & 0.5015 & 355 & 39 & 6.63 \\
\hline & 0.3 & 0.5630 & 0.4363 & 0.4916 & 405 & 41 & 5.13 \\
\hline & 0.4 & 0.5124 & 0.4510 & 0.4797 & 525 & 49 & 3.98 \\
\hline & 0.5 & 0.4973 & 0.4534 & 0.4743 & 553 & 50 & 3.73 \\
\hline & 0.6 & 0.4879 & 0.4461 & 0.4661 & 621 & 50 & 3.46 \\
\hline & 0.7 & 0.4910 & 0.4436 & 0.4661 & 664 & 46 & 3.43 \\
\hline & 0.8 & 0.4927 & 0.4436 & 0.4669 & 684 & 46 & 3.47 \\
\hline & 0.9 & 0.4949 & 0.4436 & 0.4679 & 687 & 46 & 3.49 \\
\hline \multirow[t]{9}{*}{ Krogan } & 0.1 & 0.5856 & 0.5956 & 0.5906 & 473 & 68 & 4.51 \\
\hline & 0.2 & 0.5658 & 0.5980 & 0.5815 & 509 & 67 & 4.27 \\
\hline & 0.3 & 0.5401 & 0.5980 & 0.5676 & 561 & 68 & 3.60 \\
\hline & 0.4 & 0.4888 & 0.6422 & 0.5551 & 759 & 80 & 2.86 \\
\hline & 0.5 & 0.2728 & 0.7230 & 0.3962 & 780 & 81 & 2.82 \\
\hline & 0.6 & 0.3095 & 0.7230 & 0.4335 & 835 & 83 & 2.77 \\
\hline & 0.7 & 0.2984 & 0.7230 & 0.4225 & 858 & 78 & 2.76 \\
\hline & 0.8 & 0.3090 & 0.6005 & 0.4080 & 868 & 79 & 2.79 \\
\hline & 0.9 & 0.4989 & 0.6471 & 0.5634 & 870 & 79 & 2.81 \\
\hline \multirow[t]{9}{*}{ MIPS } & 0.1 & 0.3784 & 0.5735 & 0.4559 & 703 & 46 & 3.95 \\
\hline & 0.2 & 0.3689 & 0.5760 & 0.4498 & 721 & 47 & 3.78 \\
\hline & 0.3 & 0.3375 & 0.5980 & 0.4315 & 803 & 54 & 3.10 \\
\hline & 0.4 & 0.3231 & 0.6765 & 0.4373 & 1173 & 72 & 2.33 \\
\hline & 0.5 & 0.3238 & 0.6765 & 0.4379 & 1186 & 71 & 2.32 \\
\hline & 0.6 & 0.3288 & 0.6691 & 0.4409 & 1244 & 69 & 2.31 \\
\hline & 0.7 & 0.3299 & 0.6642 & 0.4408 & 1255 & 67 & 2.32 \\
\hline & 0.8 & 0.3315 & 0.6642 & 0.4423 & 1258 & 67 & 2.32 \\
\hline & 0.9 & 0.3315 & 0.6642 & 0.4423 & 1258 & 67 & 2.33 \\
\hline
\end{tabular}
$g c$. The results are shown in Table 4 . where $P C$ is the total
Table 4 Influence of parameters gc 
number of predicted protein complexes, Perfect is the count of predicted protein complexes and standard complexes are perfectly matched, $O S(p c, s c)=1$. $A S$ represents the average size of the predicted protein complexes.

F-measure reflects the effectiveness of the algorithm, and Perfect reflects the accuracy of the algorithm. In order to comprehensively consider the impact of $g c$ on the performance of the algorithm, we performed min-max normalization on F-measure and Perfect. The parameter $F$ is defined as the harmonic mean of F-measure and Perfect, as shown in eq. (9).

$$
\text { NFmeasure }=\frac{F-\text { meausre }-\min (F-\text { measure })}{\max (F-\text { measure })-\min (F-\text { measure })}
$$

$$
\begin{aligned}
& \text { NPerfect }=\frac{\text { Perfect }-\min (\text { Perfect })}{\max (\text { Perfect })-\min (\text { Perfect })} \\
& F=\frac{\text { NFmeasure }+ \text { NPerfect }}{2}
\end{aligned}
$$

The influence of parameters $g c$ is shown in Fig. 3. $F$ value gets the best value when $g c$ equals 0.4 in DIP, Gavin and Krogan. When $g c$ is greater than 0.4 the $F$ value will rise tends to be stable in MIPS. So set $g c$ for 0.4 in this study.

\section{Comparison based on precision, recall and F-measure}

The performance of ONCQS is compared with five other state-of-the-art protein complex prediction algorithms: MCODE, MCL, CORE, ClusterONE and $\mathrm{COACH}$. The MCODE and ClusterONE are run using Cytoscape [36] and the parameters are set to the default setting. Figure 4 depicts the Precision, Recall and Fmeasure of each algorithm on four datasets. As shown in Fig. 4, it is obvious that the Recall and F-measure value of our method is much more excellent than other methods on four datasets. It indicates that ONCQS algorithm can detect protein complexes more accurately. In Fig. 4a DIP dataset, the ONCQS achieved Precision, Recall and F-measure values of $0.3561,0.8260$ and 0.4976 , respectively. The other methods MCODE, MCL, CORE, ClusterONE and $\mathrm{COACH}$ achieved F-measure values $0.0919,0.0168,0.1794,0.3690$ and 0.4270. In Fig. 4b Gavin dataset, the ONCQS achieved the highest Recall 0.4510 and F-measure 0.4797. In Fig. 4c Krogan dataset, the ONCQS achieved the highest Recall 0.6422 and Fmeasure 0.5551, which obviously outperforms other methods. In Fig. 4d, the methods MCODE,MCL, CORE, ClusterONE, COACH and ONCQS achieved F-measure values $0.1524,0.2321,0.0796,0.2755,0.3548$ and 0.4373 . Table 5 depicts the PC, Perfect and AS of each algorithm on four datasets. Obviously, the algorithm ONCQS can mine the protein complex more accurately, and the perfect value is much higher than other algorithms.

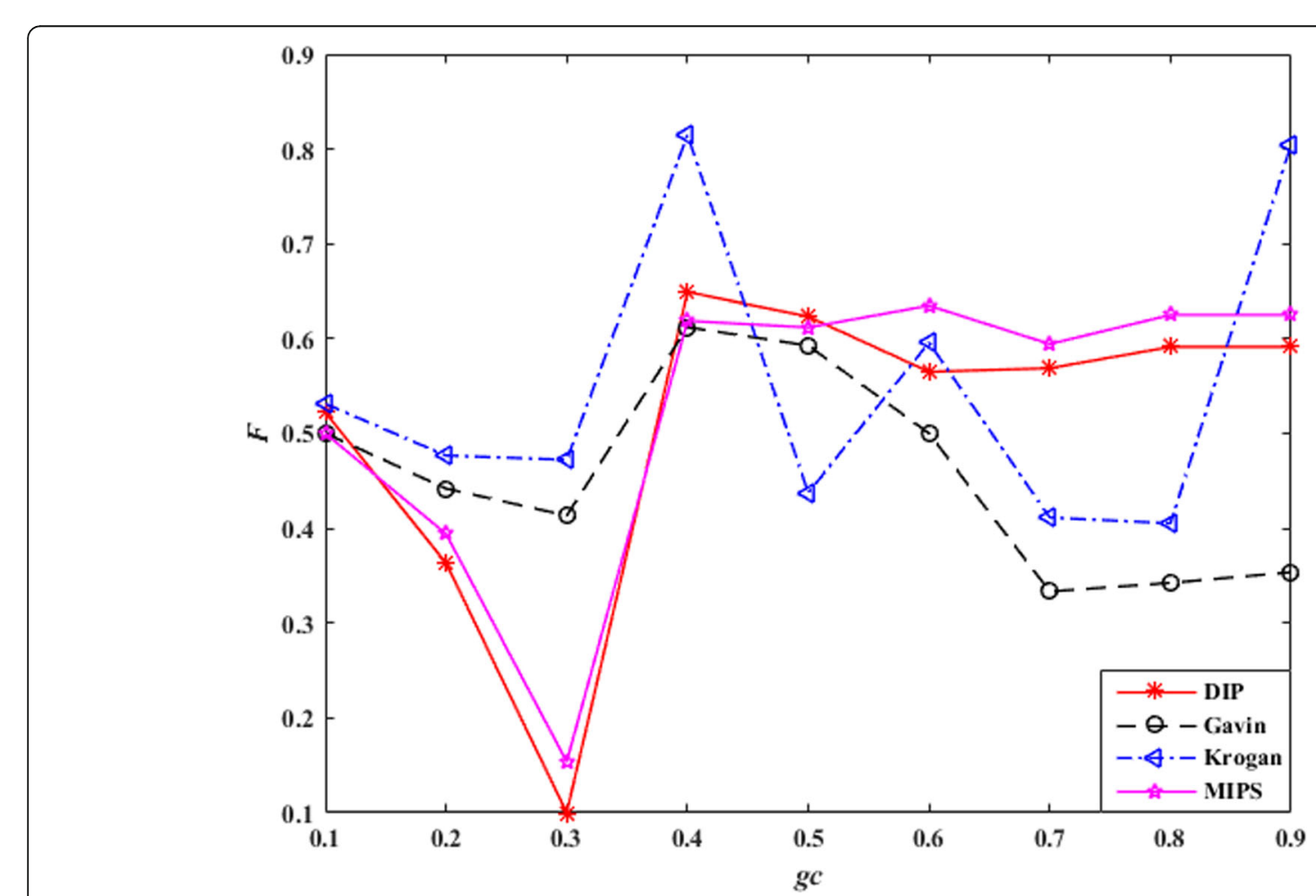

Fig. 3 Influence of parameters gc 


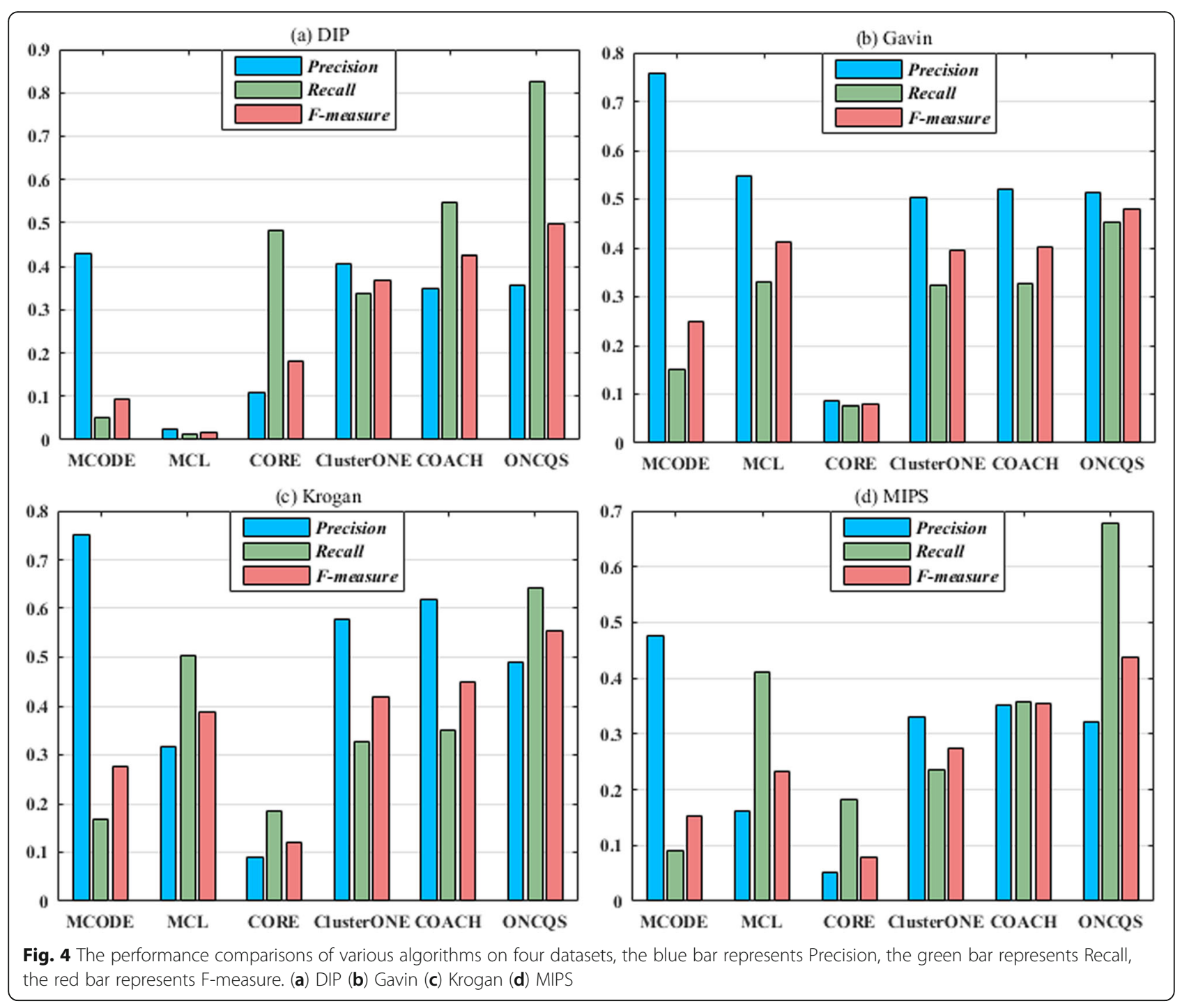

\section{Comparison with standard complexes}

In order to show the experimental results more clearly, we visualized the $379^{\text {th }}$ standard protein complex of CYC2008 "UTP B complex" and the corresponding mining results of 6 algorithm on Krogan dataset in Fig. 5. As shown in Fig. 5a, the standard protein complex is bound together by 6 proteins. Figure 5b shows the results of MCL and MCODE, the pink area is the result of the MCL algorithm, and the orange area is the result of MCODE. MCL algorithm has 2 proteins that are incorrect predictions. MCODE predicts three closely connected subgraphs into a protein complex. Figure $5 \mathrm{c}$

Table $\mathbf{5}$ The performance comparison of several typical algorithms on four datasets

\begin{tabular}{|c|c|c|c|c|c|c|c|c|c|c|c|c|}
\hline \multirow[t]{2}{*}{ Algorithms } & \multicolumn{3}{|l|}{ DIP } & \multicolumn{3}{|c|}{ Gavin } & \multicolumn{3}{|c|}{ Krogan } & \multicolumn{3}{|l|}{ MIPS } \\
\hline & $P C$ & Perfect & $A S$ & $P C$ & Perfect & $A S$ & $\overline{P C}$ & Perfect & $A S$ & $P C$ & Perfect & $A S$ \\
\hline$\overline{\mathrm{MCODE}}$ & 49 & 1 & 16.73 & 66 & 8 & 9.12 & 76 & 11 & 7.21 & 63 & 3 & 8.33 \\
\hline $\mathrm{MCL}$ & 189 & 0 & 3.76 & 217 & 20 & 6.83 & 550 & 17 & 4.63 & 922 & 12 & 4.67 \\
\hline CORE & 1707 & 6 & 3.01 & 294 & 0 & 2.58 & 820 & 0 & 2.32 & 1745 & 0 & 2.18 \\
\hline ClusterONE & 372 & 6 & 4.94 & 243 & 13 & 6.92 & 241 & 12 & 5.26 & 295 & 3 & 4.24 \\
\hline $\mathrm{COACH}$ & 899 & 16 & 8.90 & 321 & 12 & 10.18 & 355 & 17 & 7.55 & 489 & 9 & 10.31 \\
\hline ONCQS & 1640 & 103 & 2.67 & 525 & 49 & 3.98 & 759 & 80 & 2.86 & 1173 & 72 & 2.34 \\
\hline
\end{tabular}




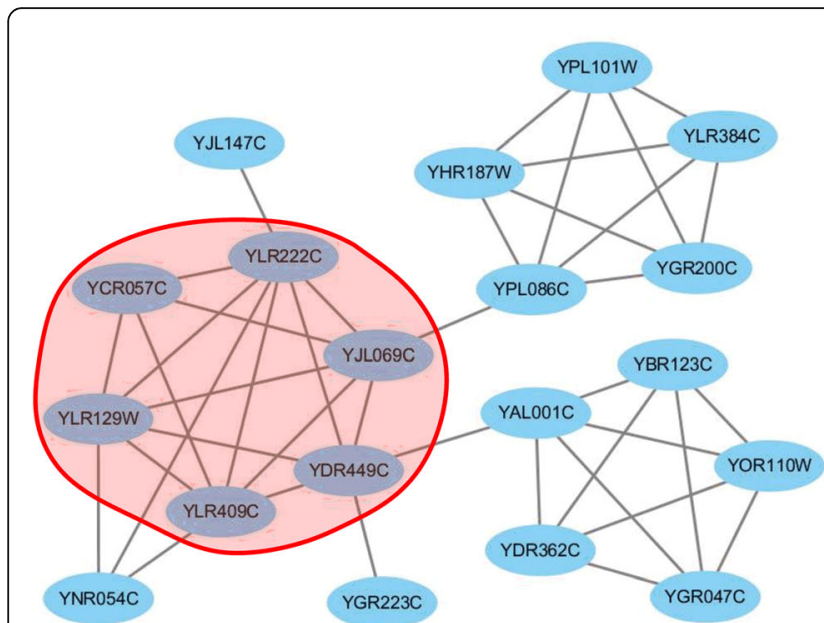

(a) Standard

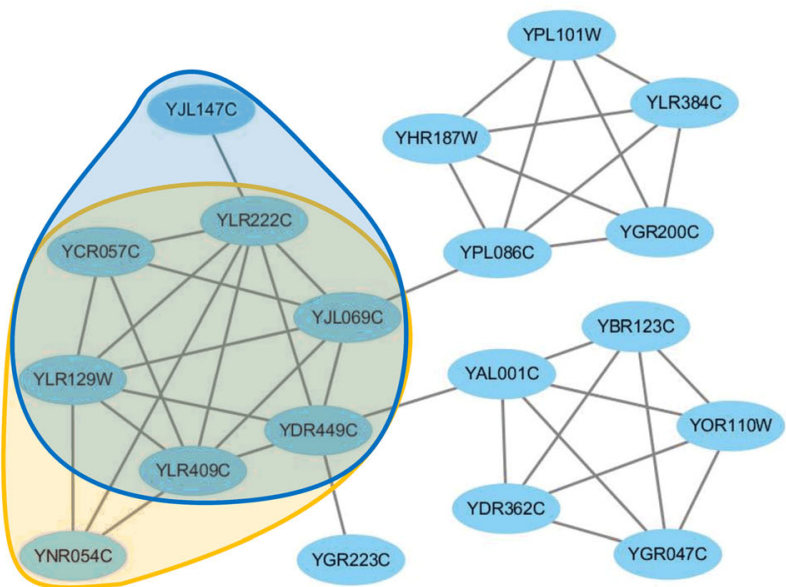

(c) ClusterONE (blue) and COACH (yellow)

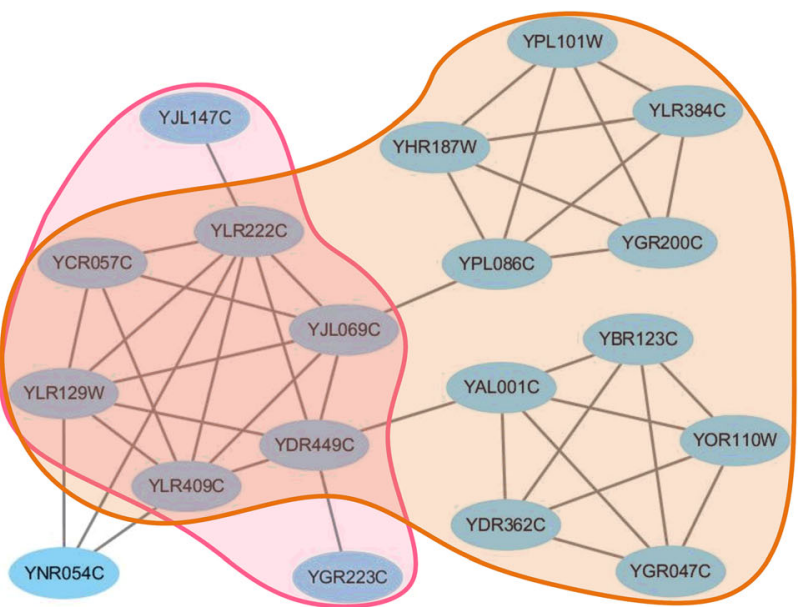

(b) MCL (pink) and MCODE (orange)

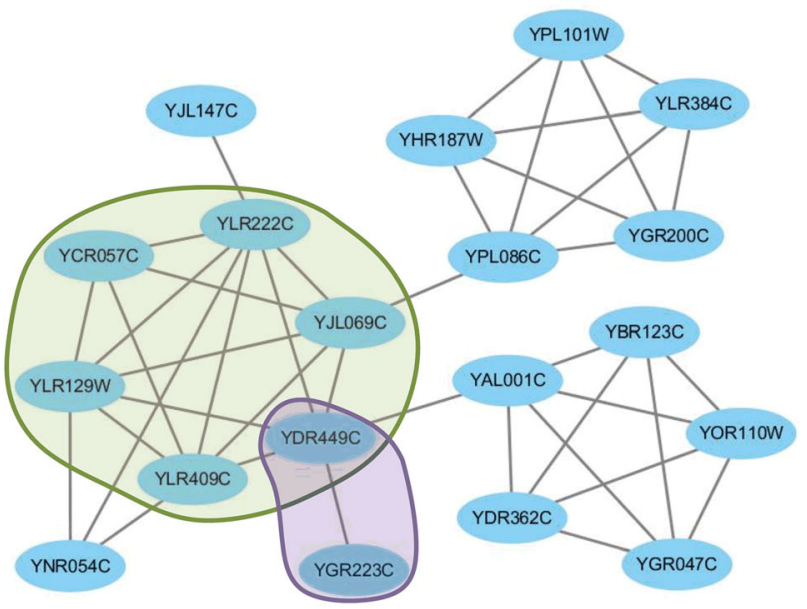

(d) ONCQS (green) and CORE (purple)

Fig. 5 Visualization of the $379^{\text {th }}$ standard protein complex of Krogan. (a) Standard (red area) (b) MCL (pink area) and MCODE (orange area) (c) ClusterONE (blue area) and COACH (yellow area) (d) ONCQS (green area) and CORE (purple area)

shows the results of ClusterONE and $\mathrm{COACH}$, the blue area is the result of the ClusterONE algorithm, and the yellow area is the result of $\mathrm{COACH}$. Both ClusterONE and $\mathrm{COACH}$ algorithms have a mispredicted protein. In Fig. $5 \mathrm{~d}$, green area and purple area are the results of ONCQS and CORE respectively. ONCQS correctly found 6 proteins. Other algorithms have erroneous prediction of proteins.

\section{Compare the ability to mine overlapping protein complexes}

Individual proteins can participate in the formation of a variety of different protein complexes, different complexes perform different functions. There are overlaps between protein complexes. ONCQS method is proposed to mine overlapping protein complexes. The standard protein complexes in the $\mathrm{CYC} 2008$ database contain many overlapping protein complexes. Figure 2 shows a pair of overlapping protein complexes elF3 complex and multi-elF complex. We analyzed the matching of the six algorithms in four databases to these two complexes. The elF3 complex and multi-elF complex were recorded as $s c 1$ and $s c 2$. Their complexes information is listed in Table 6.

The elF3 complex contains seven proteins, multi-elF complex contains eight proteins, three of which are common. Then we analyze the clustering results of the 6 algorithms in four databases respectively. Similarly, only when the overlapping score is greater than 0.2 , the matching is

Table 6 The complexes information of elF3 complex and multielF complex

\begin{tabular}{ll}
\hline elF3 complex (sc1) & multi-elF complex (sc2) \\
\hline YMR012W YLR192C YMR309C & YER025W YMR309C YOR361C \\
YOR361C YBR079C YMR146C & YNL244C YJR007W YPL237W \\
YDR429C & YMR146C YPR041W \\
\hline
\end{tabular}


Table 7 The performance comparison of mining overlapping proteins in DIP

\begin{tabular}{|c|c|c|c|c|}
\hline Algorithm & $\begin{array}{l}\text { Predicted elF3 } \\
\text { complex ( } p c 1)\end{array}$ & $\begin{array}{l}\text { OS(pcl, } \\
s c 1)\end{array}$ & $\begin{array}{l}\text { Predicted multi-elf } \\
\text { complex ( } p c 2)\end{array}$ & $\begin{array}{l}\text { OS(pc2, } \\
s c 2)\end{array}$ \\
\hline MCODE & - & - & - & - \\
\hline$M C L$ & - & - & - & - \\
\hline CORE & $\begin{array}{l}\text { YMR146C YDR429C } \\
\text { YBR079C }\end{array}$ & 0.4286 & - & - \\
\hline ClusterONE & $\begin{array}{l}\text { YPR041W YDR429C } \\
\text { YBR079C YMR309C } \\
\text { YMR146C YPL001W } \\
\text { YOR361C YDR091C } \\
\text { YLR192C YPL105C }\end{array}$ & 0.5143 & - & - \\
\hline $\mathrm{COACH}$ & $\begin{array}{l}\text { YDR429C YBR079C } \\
\text { YMR146C YMR309C } \\
\text { YNL244C YOR361C } \\
\text { YPR041W YPR086W } \\
\text { YLR192C }\end{array}$ & 0.5714 & - & - \\
\hline ONCQS & $\begin{array}{l}\text { YBR079C YDR429C } \\
\text { YLR192C YMR146C } \\
\text { YMR309C YNL244C } \\
\text { YOR361C YPR041W }\end{array}$ & 0.6429 & $\begin{array}{l}\text { YBR079C } \\
\text { YJR007W } \\
\text { YPL237W } \\
\text { YPR041W }\end{array}$ & 0.2813 \\
\hline
\end{tabular}

considered successful, and when there are multiple successful matches, the maximum overlapping score is obtained. The results of the 6 algorithms in DIP, Gavin, Krogan and MIPS are shown in Tables 7, 8, 9 and 10 respectively. Where $p c 1$ represents the predicted complex

Table 8 The performance comparison of mining overlapping proteins in Gavin

\begin{tabular}{|c|c|c|c|c|}
\hline Algorithm & $\begin{array}{l}\text { Predicted elF3 } \\
\text { complex ( } p c 1)\end{array}$ & $\begin{array}{l}\text { OS }(p c 1 \\
s c 1)\end{array}$ & $\begin{array}{l}\text { Predicted multi-elF } \\
\text { complex }(p c 2)\end{array}$ & $\begin{array}{l}O S(p c 2, \\
s c 2)\end{array}$ \\
\hline MCODE & $\begin{array}{l}\text { YDR429C YBR079C } \\
\text { YMR309C }\end{array}$ & 0.4286 & - & - \\
\hline $\mathrm{MCL}$ & $\begin{array}{l}\text { YBR079C YDR091C } \\
\text { YDR429C YLR192C } \\
\text { YMR309C } \\
\text { YPR041W YOR361C } \\
\text { YMR146C YNL244C } \\
\text { YNL096C }\end{array}$ & 0.5143 & - & - \\
\hline CORE & - & - & - & - \\
\hline ClusterONE & $\begin{array}{l}\text { YMR309C } \\
\text { YMR146C } \\
\text { YOR096W } \\
\text { YOR204W } \\
\text { YOR361C YPR041W } \\
\text { YNL096C YNL244C } \\
\text { YBR079C YDR091C }\end{array}$ & 0.4286 & - & - \\
\hline $\mathrm{COACH}$ & $\begin{array}{l}\text { YNL096C YPR041W } \\
\text { YOR361C } \\
\text { YMR146C } \\
\text { YOR204W YAL035W } \\
\text { YBR079C YDR429C } \\
\text { YLR192C YMR309C } \\
\text { YOL120C YJR123W }\end{array}$ & 0.4286 & $\begin{array}{l}\text { YNL244C YDR429C } \\
\text { YOR361C YMR146C } \\
\text { YOR204W YAL035W } \\
\text { YBR079C YLR192C } \\
\text { YMR309C YPR041W } \\
\text { YJL190C YBL072C } \\
\text { YJR123W }\end{array}$ & 0.2404 \\
\hline ONCQS & $\begin{array}{l}\text { YAL035W YBR079C } \\
\text { YDR429C YLR192C } \\
\text { YMR309C YPR041W } \\
\text { YOR361C YMR146C }\end{array}$ & 0.6429 & - & - \\
\hline
\end{tabular}

Table 9 The performance comparison of mining overlapping proteins in Krogan

\begin{tabular}{|c|c|c|c|c|}
\hline Algorithm & $\begin{array}{l}\text { Predicted elF3 } \\
\text { complex ( } p c 1)\end{array}$ & $\begin{array}{l}\text { OS(pCl, } \\
s c 1)\end{array}$ & $\begin{array}{l}\text { Predicted multi-elf } \\
\text { complex ( } p c 2 \text { ) }\end{array}$ & $\begin{array}{l}\text { OS(pc2, } \\
s c 2)\end{array}$ \\
\hline$\overline{M C O D E}$ & - & - & - & - \\
\hline $\mathrm{MCL}$ & $\begin{array}{l}\text { YBR065C YBR079C } \\
\text { YCR060W YDR047W } \\
\text { YDR408C YDR429C } \\
\text { YGL016W YHR034C } \\
\text { YMR309C YOR361C } \\
\text { YPR041W }\end{array}$ & 0.2078 & - & - \\
\hline CORE & - & - & - & - \\
\hline ClusterONE & $\begin{array}{l}\text { YOR361C YER025W } \\
\text { YMR309C YBR079C } \\
\text { YPL105C YMR146C } \\
\text { YBR065C YDR429C } \\
\text { YPR041W }\end{array}$ & 0.3968 & - & - \\
\hline $\mathrm{COACH}$ & $\begin{array}{l}\text { YMR146C YMR309C } \\
\text { YDR429C YBR065C } \\
\text { YBR079C YOR361C } \\
\text { YPR041W }\end{array}$ & 0.5102 & $\begin{array}{l}\text { YJR007W YBR079C } \\
\text { YMR146C YMR309C } \\
\text { YOR361C YPR041W } \\
\text { YER025W YDR429C }\end{array}$ & 0.5625 \\
\hline ONCQS & $\begin{array}{l}\text { YBR079C YDR429C } \\
\text { YMR146C YMR309C } \\
\text { YOR361C }\end{array}$ & 0.7143 & $\begin{array}{l}\text { YBR079C YER025W } \\
\text { YJR007W YOR361C } \\
\text { YPR041W }\end{array}$ & 0.4000 \\
\hline
\end{tabular}

that matches elF3 complex ( $s c 1), p c 2$ represents the predicted complex that matches multi-elF complex ( $s c 2)$. The boldface indicates that the proteins are predicted correctly.

As shown in Tables 7, 8, 9 and 10, MCODE, MCL, CORE and ClusterONE cannot detect overlapping protein complexes. MCODE and CORE failed to dig out complexes that match $s c 1$ and $s c 2$ respectively. $\mathrm{COACH}$ can dig out protein complexes that match $s c 1$ and $s c 2$, the accuracy is not as good as ONCQS. ONCQS achieved the best performance in identifying overlapping protein complexes. Both CluterONE and $\mathrm{COACH}$ algorithms are proposed for mining

Table 10 The performance comparison of mining overlapping proteins in MIPS

\begin{tabular}{|c|c|c|c|c|}
\hline Algorithm & $\begin{array}{l}\text { Predicted elF3 } \\
\text { complex }(p c 1)\end{array}$ & $\begin{array}{l}O S(p \subset 1 \\
s c 1)\end{array}$ & $\begin{array}{l}\text { Predicted multi-elF } \\
\text { complex ( } p c 2)\end{array}$ & $\begin{array}{l}\text { OS(pc2, } \\
s c 2)\end{array}$ \\
\hline MCODE & - & - & - & - \\
\hline$M C L$ & $\begin{array}{l}\text { YBR079C YDR429C } \\
\text { YMR146C YMR309C } \\
\text { YNL244C YOR361C } \\
\text { YPL105C YPR041W }\end{array}$ & 0.4464 & - & - \\
\hline CORE & - & - & - & - \\
\hline ClusterONE & - & - & $\begin{array}{l}\text { YPR041W YNL244C } \\
\text { YOR361C YMR146C } \\
\text { YMR309C YBR079C }\end{array}$ & 0.5208 \\
\hline $\mathrm{COACH}$ & $\begin{array}{l}\text { YMR146C YOR361C } \\
\text { YDR429C YMR309C } \\
\text { YPL105C }\end{array}$ & 0.4571 & $\begin{array}{l}\text { YMR309C YOR361C } \\
\text { YPR041W YBR079C } \\
\text { YMR146C YNL244C }\end{array}$ & 0.5208 \\
\hline ONCQS & $\begin{array}{l}\text { YDR429C YMR146C } \\
\text { YOR361C }\end{array}$ & 0.4286 & $\begin{array}{l}\text { YBR079C YMR309C } \\
\text { YNL244C YOR361C } \\
\text { YPR041W }\end{array}$ & 0.4000 \\
\hline
\end{tabular}


overlapping protein complexes. In this case, ClusterONE cannot detect overlapping protein complexes, and the performance of $\mathrm{COACH}$ is poor. This further shows that it is meaningful to design efficient and accurate algorithms to mine overlapping protein complexes. ONCQS combines GO functional annotation information, which can improve the accuracy of the algorithm.

\section{Conclusion}

Protein complexes are involved in multiple biological processes, and thus the detection of protein complexes is essential to understand cellular mechanisms. At the same time, there is overlap between protein complexes. This paper proposes a new algorithm ONCQS to identify overlapping protein complexes based on overlay network chain in quotient space. Combining the network properties of protein interaction networks with the biological properties of proteins, protein complexes are seen as nodes in the overlay network. Build an overlay network chain to mine protein complexes. Compared with the other competing clustering methods, ONCQS can effectively identify the overlapping protein complexes and has higher precision and accuracy.

\section{Abbreviations}

GO: Gene ontology; MCL: Markov clustering; ONCQS: Overlay network chain in quotient space; OS: Overlapping score; PPI: Protein-protein interaction

\section{Acknowledgments}

We would like to thank YC Zhang for providing the source code in the $\mathrm{MCL}$ method.

\section{About this supplement}

This article has been published as part of BMC Bioinformatics Volume 20 Supplement 25, 2019: Proceedings of the 2018 International Conference on Intelligent Computing (ICIC 2018) and Intelligent Computing and Biomedical Informatics (ICBI) 2018 conference: bioinformatics. The full contents of the supplement are available online at https://bmcbioinformatics.biomedcentral. com/articles/supplements/volume-20-supplement-25.

\section{Authors' contributions}

XJL initiated this study, JZ designed the algorithm, implemented the matlab code and performed the computational analysis under the supervision of $\mathrm{XJL}$. All authors wrote, read and approved the final manuscript.

\section{Funding}

The publication cost of this article has been funded by the National Natural Science Foundation of China $(61972451,61672334,61902230)$ and the Fundamental Research Funds for the Central Universities, Shaanxi Normal University (GK201901010, GK201804006).

\section{Availability of data and materials}

The methods MCODE and ClusterONE are run using Cytoscape and the parameters are set to the default setting. GO-slims data available at https:// downloads.yeastgenome.org/curation/literature/go_slim_mapping.tab.

Ethics approval and consent to participate

Not applicable.

\section{Consent for publication}

Not applicable.

\section{Competing interests}

The authors declare that they have no competing interests.

Published: 24 December 2019

\section{References}

1. Marsh J, Hernández H, Hall Z, Ahnert S, Perica T, Robinson C, Teichmann S. Protein complexes are under evolutionary selection to assemble via ordered pathways. Cell. 2013;153(2):461-70.

2. Winzeler $E A$, Shoemaker DD, Astromoff $A$, Liang $H$, Anderson $K$, Andre $B$, Bangham R, Benito R, Boeke JD, Bussey H. Functional characterization of the S. cerevisiae genome by gene deletion and parallel analysis. Science. 1999; 285(5429):901-6.

3. Ruan P, Hayashida M, Akutsu T, Vert JP. Improving prediction of heterodimeric protein complexes using combination with pairwise kernel. Bmc Bioinformatics. 2018;19(Suppl 1):39.

4. Wang J, Liang J, Zheng W, Zhao X, Mu J. Protein complex detection algorithm based on multiple topological characteristics in PPI networks. Inform Sci. 2019;489:78-92.

5. Pereira-Leal J. The evolutionary origin of protein complexes. Bmc Bioinformatics. 2005;6(Suppl 3):1-1.

6. Link AJ, Eng J, Schieltz DM, Carmack E, Mize GJ, Morris DR, Garvik BM. Direct analysis of protein complexes using mass spectrometry. Nat Biotechnol. 1999;17(7):676-82.

7. Ho Y, Gruhler A, Heilbut A, Bader GD, Moore L, Adams SL, Millar A, Taylor P, Bennett K, Boutilier K. Systematic identification of protein complexes in Saccharomyces cerevisiae by mass spectrometry. Nature. 2002;415(6868):180-3.

8. Legrain P, Wojcik J, Gauthier JM. Protein-protein interaction maps: a lead towards cellular functions. Trends Genet. 2001;17(6):346-52.

9. Bader GD, Hogue CW. An automated method for finding molecular complexes in large protein interaction networks. BMC Bioinformatics. 2003:4(1):2.

10. Gavin AC, Aloy P, Grandi P, Krause R, Boesche M, Marzioch M, Rau C, Jensen $L$, Bastuck S, Dümpelfeld B. Proteome survey reveals modularity of the yeast cell machinery. Nature. 2006;440(7084):631-6.

11. Leung HC, Xiang Q, Yiu SM, Chin FY. Predicting protein complexes from PPI data: a core-attachment approach. J Computl Biol J Comput Mol Cell Biol. 2009;16(2):133

12. Min W, Li X, Kwoh CK, Ng SK. A core-attachment based method to detect protein complexes in PPI networks. Bmc Bioinformatics. 2009:10(1):1-16.

13. Liu G, Wong L, Chua HN. Complex discovery from weighted PPI networks. Bioinformatics. 2009;25(15):1891-7.

14. Nepusz T, Yu H, Paccanaro A. Detecting overlapping protein complexes in protein-protein interaction networks. Nat Methods. 2012;9(5):471.

15. Xu B, Li K, Zheng W, Liu X, Zhang Y, Zhao Z, He Z. Protein complexes identification based on go attributed network embedding. BMC Bioinformatics. 2018;19(1):535.

16. Van Dongen S: Graph Clustering by Flow Simulation. Phd Thesis University of Utrecht 2000.

17. Lei X, Ding Y, Wu FX. Detecting protein complexes from DPINs by density based clustering with pigeon-inspired optimization algorithm. Sci China Inf Sci. 2016:59(7):070103.

18. Lei $X$, Ding $Y$, Fujita $H$, Zhang A. Identification of dynamic protein complexes based on fruit fly optimization algorithm. Knowl-Based Syst. 2016:105(C):270-7.

19. Lei X, Wang F, Wu FX, Zhang A, Pedrycz W. Protein complex identification through Markov clustering with firefly algorithm on dynamic proteinprotein interaction networks. Inform Sci. 2016;329(6):303-16.

20. Ling Z, Bo Z. Theory of fuzzy quotient space (methods of fuzzy granular computing). J Software. 2003;14(4):770-6.

21. Xu F, Zhang L, Wang $L$. Approach of the fuzzy granular computing based on the theory of quotient space. Pattern Recognit Artifl Intell. 2004;17(4):424-9.

22. Tang $\mathrm{XQ}$, Zhu P, Cheng JX. Cluster analysis based on fuzzy quotient space. J Software. 2008;19(4):861-8

23. Xenarios I, Salwínski L, Duan XJ, Higney P, Kim SM, Eisenberg D. DIP, the database of interacting proteins: a research tool for studying cellular networks of protein interactions. Nucleic Acids Res. 2002;30(1):303.

24. Krogan NJ, Cagney G, Yu H, Zhong G, Guo X, Ignatchenko A, Li J, Pu S, Datta N, Tikuisis AP. Global landscape of protein complexes in the yeast Saccharomyces cerevisiae. Nature. 2006:440(7084):637-43.

25. Güldener U, Münsterkötter M, Oesterheld M, Pagel P, Ruepp A, Mewes HW, Stümpflen V. MPact: the MIPS protein interaction resource on yeast. Nucleic Acids Res. 2006;34(Database issue):D436. 
26. Lei X, Liang J. Neighbor Affinity-based core-attachment method to detect protein complexes in dynamic PPI networks. Molecules. 2017;22(7):1223.

27. Zhao J, Lei X, Wu FX. Predicting Protein Complexes in Weighted Dynamic PPI Networks Based on ICSC. Complexity. 2017;2017:1-11.

28. Lei X, Zhang Y, Cheng S, Wu FX, Pedrycz W. Topology potential based seedgrowth method to identify protein complexes on dynamic PPI data. Inform Sci. 2018;425:140-53

29. Lei $X$, Zhao J, Fujita H, Zhang A. Predicting essential proteins based on RNASeq, subcellular localization and GO annotation datasets. Knowl-Based Syst. 2018;151:136-48.

30. Zhao J, Lei X. Mining overlapping protein complexes in PPI network based on granular computation in quotient space. In: International Conference on Intelligent Computing. Cham: Springer; 2018. p. 691-6.

31. Zhang Y, Lin H, Yang Z, Wang J, Li Y, X X B. Protein complex prediction in large ontology attributed protein-protein interaction networks. IEEE/ACM Transact Comput Biol Bioinform. 2013;10(3):729-41.

32. Zhang L, Zhang B. The structure analysis of fuzzy sets. Int J Approx Reason. 2005;40(1):92-108.

33. Zhang L, He FG, Zhang YP, Zhao S. A new algorithm for optimal path finding in complex networks based on the quotient space. Fundamenta Informaticae. 2009;93(4):459-69.

34. Fugui H, Ling Z, Yanping Z, Shu Z. Quotient space overlay model for calculating network shortest path and building method thereof; 2008.

35. Pu S, Wong J, Turner B, Cho E, Wodak SJ. Up-to-date catalogues of yeast protein complexes. Nucleic Acids Res. 2009;37(3):825.

36. Smoot ME, Ono K, Ruscheinski J, Wang PL, Ideker T. Cytoscape 2.8: new features for data integration and network visualization. Bioinformatics. 2011; 27(3):431-2.

\section{Publisher's Note}

Springer Nature remains neutral with regard to jurisdictional claims in published maps and institutional affiliations.

Ready to submit your research? Choose BMC and benefit from:

- fast, convenient online submission

- thorough peer review by experienced researchers in your field

- rapid publication on acceptance

- support for research data, including large and complex data types

- gold Open Access which fosters wider collaboration and increased citations

- maximum visibility for your research: over $100 \mathrm{M}$ website views per year

At $\mathrm{BMC}$, research is always in progress.

Learn more biomedcentral.com/submissions 